\title{
ATP16 Genes and Neighboring ORFs Are Duplicated on Chromosome IV in Saccharomyces cerevisiae
}

\author{
Masaharu Takeda (Corresponding author) \\ Department of Applied Life Science, Sojo University \\ 4-22-1 Ikeda, Kumamoto 860-0082, Japan \\ $\&$ \\ Department of Materials and Biological Engineering \\ Tsuruoka National College of Technology \\ Tsuruoka, Yamagata 997-8511, Japan \\ Tel: 81-235-25-9130Ｅ-mail: mtakeda@tsuruoka-nct.ac.jp \\ Hironori Fujioka \& Manami Shimomura \\ Department of Applied Life Science, Sojo University \\ 4-22-1 Ikeda, Kumamoto 860-0082, Japan \\ Tadashi Mabuchi \\ Department of Biochemistry \\ Interdisciplinary Graduate School of Medicine and Engineering \\ University of Yamanashi, Tamaho, Nakakoma \\ Yamanashi 409-3898, Japan \\ Atsushi Minami \\ Department of Materials and Biological Engineering \\ Tsuruoka National College of Technology \\ Tsuruoka, Yamagata 997-8511, Japan
}

Received: August 4, 2011

Accepted: August 25, $2011 \quad$ Published: December 31, 2011

doi:10.5539/jmbr.v1n1p2

URL: http://dx.doi.org/10.5539/jmbr.v1n1p2

\begin{abstract}
We present evidence that there were two closely linked copies of the ATP16 (YDL004w) gene encoding the $\delta$ subunit of $\mathrm{F}_{1} \mathrm{~F}_{0}$-ATPase complex on chromosome IV in laboratory strains, W303-1A, W303-1B, DC5, LL20, SEY2102, YPH499, and S288C of Saccharomyces cerevisiae. We previously reported that there were 2-3 copies of ATP1 $(\alpha), A T P 2(\beta)$ and ATP3 $(\gamma)$ on chromosomes II, X, and II, respectively. Homologous recombination of the ATP16 with HIS3 (YOR202w), gene walking, and long-PCR analyses showed that two ATP16 were present on the same chromosome as above genes. The gene walking estimated that the two ATP16 were separated by approximately $8.4 \mathrm{~kb}$ using ATP16 and its neighboring DNAs as probes, designated the proximal ATP16 to the telomere as ATP16a and to the distal as ATP16b. Although the nucleotide sequences of $A T P 16 a$ and $A T P 16 b$ were identical including 833 bases upstream- and 937 bases downstream of ATP16, they might be expressed differently.
\end{abstract}

Keywords: $\mathrm{F}_{1} \mathrm{~F}_{0}$-ATPase, ATP16, Repetitive genes, Chromosome IV, Saccharomyces cerevisiae 


\section{Introduction}

$\mathrm{F}_{1}$-ATPase (catalytic sector of $\mathrm{F}_{1} \mathrm{~F}_{0}$-ATPase complex, designated as $\mathrm{F}_{1}$ ) was composed of five different subunits, $\alpha, \beta, \gamma, \delta$ and $\varepsilon$ in a stoichiometry of 3: 3:1:1:1 in all aerobic cells (Cox et al.,1992; Boyer, 1993; Noji et al., 1997; Futai et al, 2000). In yeast $S$. cerevisiae, they were encoded for the nuclear genes ATP1 (YBL099w), ATP2 (YJR121w), ATP3 (YBR039w), ATP16 (YDL004w), and ATP15 (YPL271w), respectively. Recently, we had revealed that multiple copies of the ATP1 (YBL099w), ATP2 (YJR121w), and ATP3 (YBR039w) genes were arranged in tandem on each chromosome on which these genes are located (Takeda et al., 1995; Takeda et al., 1999; Takeda et al., 2010; Takeda et al., 2005; Ohnishi et al., 2003), not as reported by the Genome Project using S288C (Feldmann et al., 1994; Galibert et al., 1996; Mewes et al., 1997; Jacq et al., 1997). The copy numbers of these $F_{1} F_{0}$ subunit genes were not exactly coincident with the subunit-stoichiometry of $F_{1}$ mentioned above. In addition, these genes were repeated two or three times accompanying their several neighboring ORFs and the other tandem duplicated area on each chromosome reported by the Genome Project. Thus, the other repetitive genes such as the $\mathrm{F}_{1} \mathrm{~F}_{0}$ subunit genes might exist on the chromosomes more than we have expected.

The ATP16 (YDL004w, Giraud \& Velours, 1994) gene encoding for the $\mathrm{F}_{1} \delta$ subunit of $S$. cerevisiae was present on the left arm close to the centromere sequence (CEN4) of chromosome IV just one copy was reported by the Genome Project (Jacq et al., 1997). We showed here that two copies of the ATP16 (YDL004w) gene were also arranged in tandem accompanying the neighboring ORFs and the other tandem duplicated area on chromosome IV.

\section{Materials and Methods}

Yeast strains: The yeast strains used in this study were Saccharomyces cerevisiae DC5 (MATa leu2-3 leu2-112 his 3 can1-11), LL20 (MATa leu2 his3), W301-1A (MATa leu2-3 leu2-112 his3-11 his3-15 trp1-1 ura3-1 ade2-1 can1-100), W303-1B (MATa leu2-3 leu2-112 his3-11 his3-15 trp1-1 ura3-1 ade2-1 can1-100), YPH499 (MATa ade2 his3 leu2 trp1 ura3 lys2), and S288C (MATa SUC2 mal mel gal2 CUP1).

E. coli strains: Strains, Sure (el4- (McrA-) $\Delta(m c r C B-h s d S M R-m r r) 171$ endA1 supE44 thi-1 gyrA96 relA1 lac recBrecJ sbcC umuC::Tn5 (Kan $\left.{ }^{r}\right)$ uvrC[F' proAB lacl ${ }^{q} Z \Delta M 15$ Tn10 (Tet ${ }^{\mathrm{r}}$ )] and DH10B (F mcrA $\triangle($ mrr-hsdRMS-mrcBC) $\Phi 80 d l a c Z 4 M 15$ AlacX74 deoR recAl endA1 araD139, 4 (ara, leu)7697 galU galK rp nup $G)$, were used in this study.

Plasmids: The ATP16 gene disrupted plasmid, pYEF-16/4, was constructed as follows. The ATP16 gene was inserted into the SphI-EcoRI site of the pAML20 (Yep-type vector, Iha \& Tsurugi, 1998) and constructed the pYEATP16. The pYEATP16 was digested with Ball, and the BamHI fragment of the HIS3 gene (fill-in) was inserted into the BalI site of ATP16.

Media: E. coli carrying plasmids were grown in LB $(0.5 \%$ yeast extract, $1 \%$ bacto-tryptone, $1 \% \mathrm{NaCl})$ containing $50 \mu \mathrm{g}$ of ampicillin per ml. Yeast strains were grown on YPG (1\% yeast extract, $2 \%$ bacto-peptone, $3 \%$ glycerol), YPDM (1\% yeast extract, $0.5 \%$ bacto-peptone, $0.1 \%\left(\mathrm{NH}_{4}\right)_{2} \mathrm{SO}_{4}, 0.2 \% \mathrm{KH}_{2} \mathrm{PO}_{4}, 0.1 \% \mathrm{MgSO}_{4}$, $0.8 \%$ glucose $)$, or $\mathrm{SD}(0.67 \%$ yeast nitrogen base without amino acids, $2 \%$ glucose, and appropriate nutrients). The solid medium contained $2 \%$ agar.

Polymerase chain reaction (PCR): Long-PCR analysis was performed according to the procedure recommended for the Takara Pyrobest ${ }^{\mathrm{TM}}$ polymerase PCR kit (Takara Shuzo Co., Ltd, Osaka, Japan). The primer pairs used were 5'-TATAACTGTAGCCGTGAGGA-3' (sense) and 5'-AGTTCTAGAATGCGTACCAT-3' (antisense). These primers were located just inside the ATP16-coding region (Takeda et al., 1999; Takeda et al., 2010; Ohnishi et al., 2003; Barnes, 1994). DNA was amplified in a PCR processor (Astec, Program TEMP Control System PC-700, Fukuoka, Japan) using 30 cycles. Yeast whole DNAs from each strain used in the experiments were made by the method previously reported (Takeda et al., 1995; Takeda et al., 1999). The PCR products (ATP16a and ATP16b, respectively) from each template were cloned into a vector pBluescript (Stratagene, La Jolla, CA) for sequencing according to the procedure recommended for the Takara Blunting Kination Ligation kit (Takara).

DNA sequencing: Nucleotide sequencing was performed by the standard dideoxy chain termination method with the ABI models 373 and 310, and LI-COR model 4200L-2 sequencers.

Pulse-field gel electrophoresis: The amplified DNAs were separated in a $1 \%$ agarose $(\mathrm{w} / \mathrm{v})$ gel on an alternating CHEF gel apparatus (Bio-Rad, CA). Electrophoresis was performed for $16 \mathrm{~h}$ in $0.5 \mathrm{x}$ TBE buffer at $200 \mathrm{~V}\left(14^{\circ} \mathrm{C}\right)$ with a 2.8 to 3.4 s linear gradient, as described previously (Takeda et al., 1995; Takeda et al., 1999; Takeda et al., 2010; Takeda et al., 2005; Ohnishi et al., 2003).

Preparation of mitochondria: Cells were grown in $50 \mathrm{ml}$ of YPDM medium. After incubation for $24 \mathrm{~h}$ at $30^{\circ} \mathrm{C}$, 
the cells (ca. $2 \sim 4 \times 10^{6}$ cells $/ \mathrm{ml}$ ) were harvested and mitochondria were prepared according to the published methods (Daum et al., 1982).

Western blotting: Immunodetection analysis of proteins was performed according to the procedures described previously (Mabuchi et al., 2000).

$R T$-PCR: Single colony of each yeast line was inoculated into $10 \mathrm{ml}$ of liquid YPD medium and shake for 42 hours at $30^{\circ} \mathrm{C}$. The yeast cells were collected by centrifugation and washed. The cell pellet was suspended in 0.3 $\mathrm{ml}$ of the lysis buffer $(0.2 \mathrm{M}$ Tris- $\mathrm{HCl} \mathrm{pH} 7.5,0.5 \mathrm{M} \mathrm{NaCl}, 10 \mathrm{mM}$ EDTA, $1 \%$ SDS $)$ and transfer to a microtube carrying $0.6 \mathrm{~g}$ of $0.5 \mathrm{~mm}$ diameter glass beads (fujistone No, 06, Fuji Rikakogyo, Osaka) and $0.3 \mathrm{ml}$ of PCI (phenol/chloroform/isoamyl alcohol). The cell was disrupted by voltex for $2.5 \mathrm{~min}$. After centrifugation and alcohol precipitation, the total RNA solution was treated with Dnase (Dnase RQ, Promega) and then purified by PCI. Extraction. The total RNA ( $5 \mu \mathrm{g})$ was reverse-transcribed with Superscript III first strand cDNA synthesis system (Invitrogen) using a gene-specific reverse primer (primer c). The cDNA equivalent to $0.5 \mu \mathrm{g}$ of the total RNA was amplified with Takara Ex Taq HS (Takara) using primers for ATP16 (primers a \& b). The PCR cycle was 30 cycles of $98^{\circ} \mathrm{C}$ for $10 \mathrm{sec}$., $50^{\circ} \mathrm{C}$ for $30 \mathrm{sec}$. and $72^{\circ} \mathrm{C}$ for $1 \mathrm{~min}$.

Miscellaneous: Southern hybridization of DIG-labeled ATP16 (YDL004w, $1.0 \mathrm{~kb}$ HincII fragment), HIS3 (1.7 kb $B a m \mathrm{HI}$ fragment), and other ATP16-neighboring probes used in the experiments were performed as described previously (Takeda et al., 1995).

\section{Results}

\subsection{Disruption of ATP16 on the left arm of chromosome IV}

The ATP16 (YDL004w) gene encoding the $\mathrm{F}_{1} \delta$ subunit was mapped on the left arm close to the centromere of chromosome IV in S. cerevisiae (Jacq et al., 1997; Giraud \& Velours, 1994). The ATP16 (YDL004w) gene was disrupted with the yeast HIS3 gene (plasmid pYEF-16/4) by the homologous recombination methods as described previously (Takeda et al., 1995; Takeda et al., 1999; Takeda et al., 2010; Takeda et al., 2005; Ohnishi et al., 2003). The total DNAs (genomic DNAs) of the ATP16-disruptants derived from four different laboratory strains, DC5, LL20, W303-1A, and W303-1B, were digested with HincII, transferred on membranes, and then hybridized successively with DIG-labeled (a) ATP16 and (b) yeast HIS3 as probes. The DNAs of the four different strains gave the same hybridization patterns when the DNA were digested with various restriction enzymes, EcoRI, BamHI, HindIII, SalI, PvuII, PstI, XbaI, NcoI, and SphI. In the gene disruption experiments, the undisrupted ATP16 (YDL004w, $1.0 \mathrm{~kb}$ ) was detected in all laboratory strains used in this study, but some $\mathrm{His}^{+}$-transformants yielded undisrupted $A T P 16(\mathrm{YDL} 004 \mathrm{w}, 1.0 \mathrm{~kb})$ in addition to the disrupted ATP16 with the HIS3 gene (atp16::HIS3, $2.8 \mathrm{~kb})$. Lane 1 (W303-1B, WT) was detected the undisrupted ATP16, lanes 2-4 (transformants W30, W3, W202) were detected both undisrupted- (1.0 kb) and HIS3-disrupted- ATP16 (2.8 kb). Lanes 5 and 6 (transformants W19 and W6) were only detected HIS3-disrupted-ATP16 (Figure 1).

To confirm the occurrence of gene disruption on chromosome IV, Southern blot analysis of the chromosome were performed using DIG-ATP16 and yeast HIS3 as probes (Figure 2). The results of the Southern analysis showed that both the undisrupted-ATP16 and the disrupted-ATP16 with yeast HIS3 were mapped on chromosome IV, as described in the Genome Project. The yeast HIS3 gene was essentially present on not only chromosome XV, but also on chromosome IV located the ATP16. The results indicated that both undisrupted ATP16 and disrupted ATP16 were present on chromosome IV, i.e., the two closely-linked copies of the ATP16 (YDL004w) gene were arranged on chromosome IV as were those of ATP3 in the yeast $S$. cerevisiae (YBR039w, Ohnishi et al., 2003). We designated the two copies as ATP16a (proximal to the telomere) and ATP16b (distal to the telomere).

Most of the transformants which were co-exiting with the undisrupted-ATP16 (ATP16b) and the disrupted-ATP16 (atp16a::HIS3) were able to grow on glycerol, but the growth was not the same as those of laboratory strains (transformant W30, Figure 3-2). In contrast, few transformants were co-existed both undisrupted-ATP16 and disrupted-ATP16 (transformant W202, ATP16a, atp16b::HIS3, Figure 3-3) could not grow on glycerol as both ATP16s-disruptant (transformant W19, atp16a::HIS3, atp16b::HIS3, Figure 3-4). The ATP16b-disruptant (transformant W202, Figure 3-3) and the double ATP16s-disruptant (transformant W19, Figure 3-4) did not recover the glycerol-grown phenotype by the introduction of the wild-type ATP16.

\subsection{Gene walking analysis of two copies of ATP16}

Southern blot hybridization of purified genomic DNA from S288C and other laboratory strains were performed using the same probe (DIG-labeled ATP16). Restriction enzymes, BamHI, ClaI, KpnI, XhoI, NdeI, SphI, $S p h \mathrm{I} / \mathrm{NcoI}$, and $X b a \mathrm{I}$ were used in the experiments (Figure 4). In $S p h \mathrm{I}$ digestion (Figure 4a, lane 6), two bands 
( 8.4 and $30.4 \mathrm{~kb}$ ) were hybridized with the ATP16 probe. According to the data of the Genome Project, the long DNA band $(30.4 \mathrm{~kb})$ was expected from S288C but not the short band $(8.4 \mathrm{~kb})$. This showed that one extra SphI site, which had not been reported by the Genome Project, should be present between two closely linked gene copies of ATP16s (data not shown). Ndel (lane 5) and Xbal (lane 8) were also detected two bands used ATP16 gene as a probe.

It also means that two closely linked copies, of ATP16 (YDL004w) are located on the left arm of chromosome IV, as described for ATP1 (YBL099w, Takeda et al., 1995; Takeda et al., 1999; Takeda et al., 2010), ATP2 (YJR121w, Takeda et al., 2005), and ATP3 (YBR039w, Ohnishi et al., 2003) in the yeast S. cerevisiae.

\subsection{Map of two gene copies of ATP16 on the left arm of chromosome IV}

Long-PCR analysis using genomic DNA from the laboratory strains and a primer set of the starting and terminal codon just inside the ATP16 (YDL004w) gene were performed in order to amplify the DNA region between the two ATP16s as described previously (Takeda et al., 1999; Takeda et al., 2005; Ohnishi et al., 2003). The results showed that only approximately $8.4-\mathrm{kb}$ of PCR product were obtained (data not shown). The 8.4-kb of PCR product was hybridized with the probe of the DIG-labeled ATP16 (YDL004w), but not hybridized with the DIG-labeled CEN4 (localized between YDL001w and YDR001c on chromosome IV, data not shown). From the analysis by chromosome walking of the total (genomic) DNA of the laboratory strains and the PCR experiments, this 8.4-kb PCR product might contain the DNA region between the two ATP16s, ATP16a, and ATP16b on chromosome IV.

To confirm more detail whether this 8.4-kb PCR product was the DNA region between ATP16a and ATP16b on chromosome IV, and to identify the repeating region of the ATP16 (YDL004w) gene. The long-PCR product was hybridized with the DIG-labeled probes neighboring genes of chromosome IV In contrast, probes containing both CEN4 localized between YDL007w (RPT2) and YDL001w to YDR001c (NTH1) of chromosome IV were not hybridized respectively to the PCR product, i.e., they are located outside the repeating region (data not shown).

\subsection{Base sequence of ATP16a and ATP16b}

To determine the nucleotide sequence of $A T P 16 a$ and $A T P 16 b$, we performed the standard dideoxy chain-termination methods using the genomic DNA isolated from laboratory strains DC5, LL20, W303-1A, W303-1B, YPH499, and strain S288C, which was used in the Yeast Genome Project. As a result, no difference was detected between the two closely linked copies of ATP16s, ATP16a, and ATP16b, not only in the coding region, but also 5'-end 833 bases upstream of ATG and 3'-end 937 bases downstream from the terminal codon. They were completely identical with the nucleotide sequences registered both at the SGD (http://www.yeastgenome.org/) and at the Mips (http://mips.gsf.de/) of the ATP16 (YDL004w) and the neighboring ORFs (data not shown). Nevertheless, they seemed to function differently. In other words, the ATP16a-disruptant could grow on the glycerol medium, but the ATP16b-disruptant could not.

\subsection{Transcripts of ATP16a and ATP16b}

To verify the expression of both the ATP16a and the ATP16b, RT-PCR experiments were performed using sense (primer a) and anti-sense primers (primers b and c) as the lower panel of Figure 6. The results of the RT-PCR experiments were shown in the upper panel of Figure 6. Clearly both the ATP16a and the ATP16b were expressed. The disruptant of both the ATP16a and the ATP16b (lane 4, transformant W19) could not observed any bands (products). The transcript of the ATP16a-disruptant (lane 2, transformant W30) deeper than that of the $A T P 16 b$-disruptant (lane 3, transformant W202), i.e., the ATP16b might be expressed more than the ATP16a (Figure 6). In the preliminary experiments, the ATP16b-disruptant was easy to mutate the mtDNA. The difference of the copy-specific disruptant might be come from the difference of the conformation of the ATP16a or the $A T P 16 b$ on chromosome. The detailed experiments were under the progress.

\section{Discussion}

The $\mathrm{F}_{1} \delta$ subunit gene, ATP16 was repetitive on the left arm of chromosome IV in S. cerevisiae. Two closely linked copies of the ATP16s, the ATP16a, and the ATP16b were present near the CEN4 localized between YDL001w and YDR001c of chromosome IV. The nucleotide sequences and the deduced amino acid sequences of the $A T P 16 a$ and the $A T P 16 b$ were the same as those of two copies of ATP3 (YBR039w, ref. 9). Neither ATP16-disruptant ( $\triangle a t p 16 a, \triangle a t p 16 b)$ could grow on glycerol. It was easy to eliminate the mtDNA that both the Southern blot analyses of the two ATP16-disruptants using DIG-labeled wild-type-mtDNA and the glycerol-grown phenotype of the diploid strain with that of the $\rho^{0}$-tester strain confirmed (data not shown). Co-existing transformants of the disrupted-ATP16 and the undisrupted-ATP16 gene showed two different 
phenotypes. That is, the ATP16a-disruptant ( $\triangle a t p 16 a, A T P 16 b$, transformant W30) could grow on glycerol, although the level of its growth was less than that of the laboratory strain (ATP16a, ATP16b, WT). When the wild-type ATP16 gene was introduced into the ATP16a-disruptant, the growth on glycerol recovered to the level of that of the laboratory strain. In contrast, the ATP16b-disruptant (ATP16a, $\triangle a t p 16 b$, transformant W202) could not grow on glycerol and easily mutated mtDNA as did all ATP16-disruptants ( $\triangle a t p 16 a, \triangle a t p 16 b$, transformant W19) (data not shown). Nonetheless, both the nucleotide and the deduced amino acid sequences were apparently the same, containing 5'-, and 3'-non-coding regions of the ATP16a and the ATP16b. From these observations, the two ATP16s might be expressed differentially as in the cases of the two ATP3s (Ohnishi et al., 2003).

Although no difference in base sequence between the two ATP3s or two ATP16s was observed, most of the $A T P 3$-, or ATP16-disruption occurred in the ATP $3 a$ or the ATP16a, which is located proximal to the left telomere in comparison with that of the $A T P 3 b$ or the $A T P 16 b$, which was located distal to the left telomere in all strains used in the experiments.

The reasons for the presence of two closely linked copies such as the ATP3s and the ATP16s, which had different functions, were not yet known. However, some possibilities could be considered. First, the regulatory region (promoter) might be located farther than 833 bases upstream of the ATG-starting codon of ATP16. Second, the expression time or conditions of $A T P 16 a$ and $A T P 16 b$ might be different; there might be some difference in the higher-ordered structure of chromosome, such as the nucleosome structure of two ATP16s on the chromosome between these two ATP16s (Kawasaki et al., 2004; Krogan et al., 2004). Third, some trans-regulated elements of the two ATP3s or ATP16s gene copies might be expressed in cells such as in the GAL genes (Kodama et al., 2003) and the lipogenic enzyme genes (Stoekman et al., 2004). The regulatory regions of most yeast genes are usually located within several hundred bases upstream of the ATG (Basehoar et al., 2004; Ferrer-Martinez et al., 2004), so the probability of the first explanation ought to be low. The second or third explanations could be more feasible.

Of the five gene subunits $(\alpha, \beta, \gamma, \delta, \varepsilon)$ in $\mathrm{F}_{1}$, four subunits were repetitive accompanying their neighboring ORFs, although the repeating unit on each chromosome was different for each ATP gene. $F_{1} \varepsilon$ subunit gene, ATP15 (YPL271w), was also repetitive on chromosome XVI (manuscript in preparation). In addition, unpublished results showed that of the $\mathrm{F}_{0}$-sector in the $\mathrm{F}_{1} \mathrm{~F}_{0}$, the nuclear-coded ATP4 (YPL078c), ATP5 (YDR298c) and ATP7 (YKL016c) genes were also repetitive as other $\mathrm{F}_{1} \mathrm{~F}_{0}$ subunit genes, ATP1, ATP2, and $A T P 3$, in $S$. cerevisiae (manuscript in preparation). Thus, all $\mathrm{F}_{1}$ subunit genes and 3 nuclear-coded- $\mathrm{F}_{0}$ subunit genes were repetitive, and other genes neighboring these $F_{1} F_{0}$ subunit genes were also present in duplicate or triplicate in $S$. cerevisiae. These repetitive genes expressed and functioned, so they probably had some biological meaning(s) and played some biological role(s). The $F_{1} F_{0}$ was one of essential enzymes in eukaryotic cells to maintain the life, so the subunit genes of the complex might be necessarily maintained even if some of subunit genes become disrupted. Also, the expression of the genes might be sophisticatedly regulated using multiple-copies of the genes to produce ATP (Takeda \& Nakahara, 2009; Nakahara \& Takeda, 2010a; Nakahara \& Takeda, 2010b). In other words, the nuclear-coded subunit genes in $\mathrm{F}_{1} \mathrm{~F}_{0}$ should participate in ATP synthesis. We did not yet know precisely whether the gene repetition was confined to the area of $F_{1} F_{0}$ subunit genes on each chromosome. The physiological meaning of these multiple genes and other regions containing repeated units was still unknown. However, we needed continue to consider them in order to understand why living cells harbor gene repetition on chromosomes.

The $\delta$ subunit played in an important role of $F_{1}$ and cell death in eukaryotic cells (Matsuyama et al., 1998). In bacteria and bovine $F_{1}$-ATPase analyses, the $\delta$ subunit located and interacted with the $\alpha, \beta$ and $\gamma$ subunits (Duvezin-Caubet, 2003). However, details of the role of the $\delta$ subunit of yeast $F_{1}$ were not known, although the $\delta$ subunit was a member of the $F_{1} F_{0}$ and plays an important role in energy-transduction machinery. Certainly $\alpha, \beta$. and $\gamma$ were important in the structure and function of the complex, but other subunits $\delta$ and $\varepsilon$ might be necessary for the control of the complex, and interact with other proteins and factors. Unfortunately, little was known about the small subunits of the $F_{1}$.

As mentioned above, in aerobic cells, ATP was the energy source of biological phenomena, and the $\mathrm{F}_{1} \mathrm{~F}_{0}$ was an important enzyme in the production of ATP. The multiple subunit genes on each chromosome might regulate in some way gene expression and assembly for functions based on the combination and organization of these multiple $\mathrm{F}_{1} \mathrm{~F}_{0}$ subunit genes on each chromosome by e.g., aging, the cell division-cycle, and environmental interactions. It might not be so unusual that a protein plays different roles in living cells. We did not know still what different roles or the minute regulation to the biological phenomena were played by the two closely linked copies of the ATP16 genes in S. cerevisiae, but understanding their roles would contribute to a better understanding of living cells (Dujon et al., 2004). 


\section{References}

Barnes, W. M. (1994). PCR amplification of up to $35 \mathrm{~kb}$ DNA with high fidelity and high yield from 1 bacteriophage templates. Proc. Natl. Acad. Sci. USA, 91, 2216-2220. http://dx.doi.org/10.1073/pnas.91.6.2216

Basehoar, A. D. Zanton, S. J., \& Pugh, B.F . (2004). Identification and distinct regulation of yeast TATA box-containing genes. Cell, 116, 699-709. http://dx.doi.org/10.1016/S0092-8674(04)00205-3

Boyer, P. D. (1993). The binding change mechanism for ATP synthase- Some probabilities and possibilities. Biochim. Biophys. Acta, 1140, 215-250. http://dx.doi.org/10.1016/0005-2728(93)90063-L

Cox, G. B. et al. (1992). The structure and assembly of ATP synthase. In Ernster L Ed. In Molecular Mechanism in Bioenergetics, Ernster L (ed.,). Elsevier, 283-315.

Daum, G., Böhni P. C., \& Schatz G. (1982). Import of proteins into mitochondria. J. Biol. Chem, 257, 13028-13033.

Dujon, B. et al. (2004). Genome evolution in yeasts. Nature, 430, 35-44. http://dx.doi.org/10.1038/nature02579

Duvezin-Caubet, S. et al. (2003). The two rotor components of yeast mitochondrial ATP synthase are mechanically coupled by subunit $\delta$. Proc. Natl. Acad. Sci. U.S.A., 100, 13235-13240. http://dx.doi.org/10.1073/pnas.2135169100

Feldmann, H. et al. (1994). Complete DNA sequence of yeast chromosome II. EMBO J., 13, 5795-5809.

Ferrer-Martinez, A. et al. (2004). A glucose response element from the $S$. cerevisiae hexose transporter HXT1 gene is sensitive to glucose in human fibroblast. J. Mol. Biol, 338, 657-667. http://dx.doi.org/10.1016/j.jmb.2004.03.036

Futai, M. et al. (2000). Synthase $\left(\mathrm{H}^{+}\right.$-ATPase) coupling between catalysis, mechanical work, and proton translocation. Biochim. Biophys. Acta, 1458, 276-288. http://dx.doi.org/10.1016/S0005-2728(00)00080-3

Galibert, F. et al. (1996). Complete nucleotide sequence of Saccharomyces cerevisiae chromosome X. EMBO J, 15, 2031-2049.

Giraud, M-F. \& Velours, J. (1994). ATP synthase of yeast mitochondria: isolation of the $\mathrm{F}_{1} \delta$ subunit, sequence and disruption of the structural gene. Eur. J. Biochem, 22, 851-859. http://dx.doi.org/10.1111/j.1432-1033.1994.tb18932.x

Iha, H. \& Tsurugi, K. (1998). Shuttle-Vector System for Saccharomyces cerevisiae designed to produce C-terminal-myc-tagged fusion proteins. Biotechniques, 25, 936-938.

Jacq, C. et al. (1997). The nucleotide sequence of Saccharomyces cerevisiae chromosome IV. Nature, 387, 75-78.

Kawasaki, H., Wadhwa, R., \& Taira, K. (2004). World of small RNAs: from ribozymes to siRNA and miRNA. Differentiation, 72, 58-64. http://dx.doi.org/10.1111/j.1432-0436.2004.07202006.x

Kodama, T. et al. (2003). Unique distribution of GAL genes on chromosome XI in the yeast Saccharomyces naganishii. Curr. Microbiol, 47, 497-500. http://dx.doi.org/10.1007/s00284-003-4083-5

Krogan, N. J. et al. (2004). High-definition macromolecular composition of yeast RNA-processing complexes. Mol. Cell, 13, 225-239. http://dx.doi.org/10.1016/S1097-2765(04)00003-6

Mabuchi, T. et al. (2000). ASC1/RAS2 suppresses the growth defect on glycerol caused by the atp1-2 mutation in the yeast Saccharomyces cerevisiae. J. Biol. Chem, 275, 10492-10497. http://dx.doi.org/10.1074/jbc.275.14.10492

Matsuyama, S. et al. (1998). The mitochondrial $\mathrm{F}_{0} \mathrm{~F}_{1}$-ATPase proton pump is required for function of the proapoptotic protein Bax in yeast and mammalian cells. Mol. Cell, 1, 327-336. http://dx.doi.org/10.1016/S1097-2765(00)80033-7

Mewes, H. W. et al. (1997). Overview of the yeast genome. Nature, 387, 7-65. http://dx.doi.org/10.1038/42755

Nakahara, M. \& Takeda, M. (2010a). Characterization of the sequence spectrum of DNA based on the appearance frequency of the nucleotide sequences of the genome $-\mathrm{A}$ new method for analysis of genome structure-. J. Biomed. Sci. Eng, 3, 340-350. http://dx.doi.org/10.4236/jbise.2010.34047

Nakahara, M. \& Takeda, M. (2010b). Identification of the Interactive Region by the Homology of the Sequence Spectrum. J. Biomed. Sci. Eng, 3, 868-883. http://dx.doi.org/10.4236/jbise.2010.39117

Noji, H. et al. (1997). Direct observation of the rotation of $F_{1}$-ATPase. Nature 1997; 386: 299-302. 1997. Direct 
observation of the rotation of $\mathrm{F}_{1}$-ATPase. Nature, 386, 299-302. http://dx.doi.org/10.1038/386299a0

Ohnishi, K. et al. (2003). Studies on the ATP3 gene of Saccharomyces cerevisiae: presence of two closely linked copies, $A T P 3 a$ and $A T P 3 b$, on the right arm of chromosome II. Yeast, 20, 943-954. http://dx.doi.org/10.1002/yea.1009

Stoekman, A. K. et al. (2004). Mix is the functional heteromeric partner of the carbohydrate response element-binding protein in glucose regulation of lipogenic enzyme gene. J. Biol. Chem, 279, 15662-15669. http://dx.doi.org/10.1074/jbc.M311301200

Takeda, M. \& Nakahara, M. (2009). Structural Features of the Nucleotide Sequences of Genomes. J. Comput. Aided Chem, 10, 38-52. http://dx.doi.org/10.2751/jcac.10.38

Takeda, M. et al. (1995). Three ATP1 genes are present on chromosome II in Saccharomyces cerevisiae. J. Biochem (Tokyo), 118, 607-613.

Takeda, M. et al. (1999). The three copies of ATP1 gene are arranged in tandem on chromosome II of the yeast

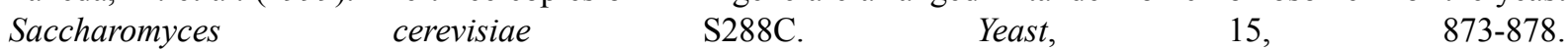
http://dx.doi.org/10.1002/(SICI)1097-0061(199907)15:10A<873::AID-YEA427>3.0.CO;2-A

Takeda, M. et al.. (2005). The three copies of ATP2 gene are arranged in tandem on chromosome X of yeast Saccharomyces cerevisiae. Curr Genet, 47, 265-272. http://dx.doi.org/10.1007/s00294-005-0565-5

Takeda, M., Nakamura, S., \& Matsushita, S. (2010). Genome organization of the three identical ATP1 genes on the left arm of chromosome II of Saccharomyces cerevisiae.- Sequence analysis of the 35-kb region containing three ATP1 genes. The Open Genomics Journal, 3, 9-17.
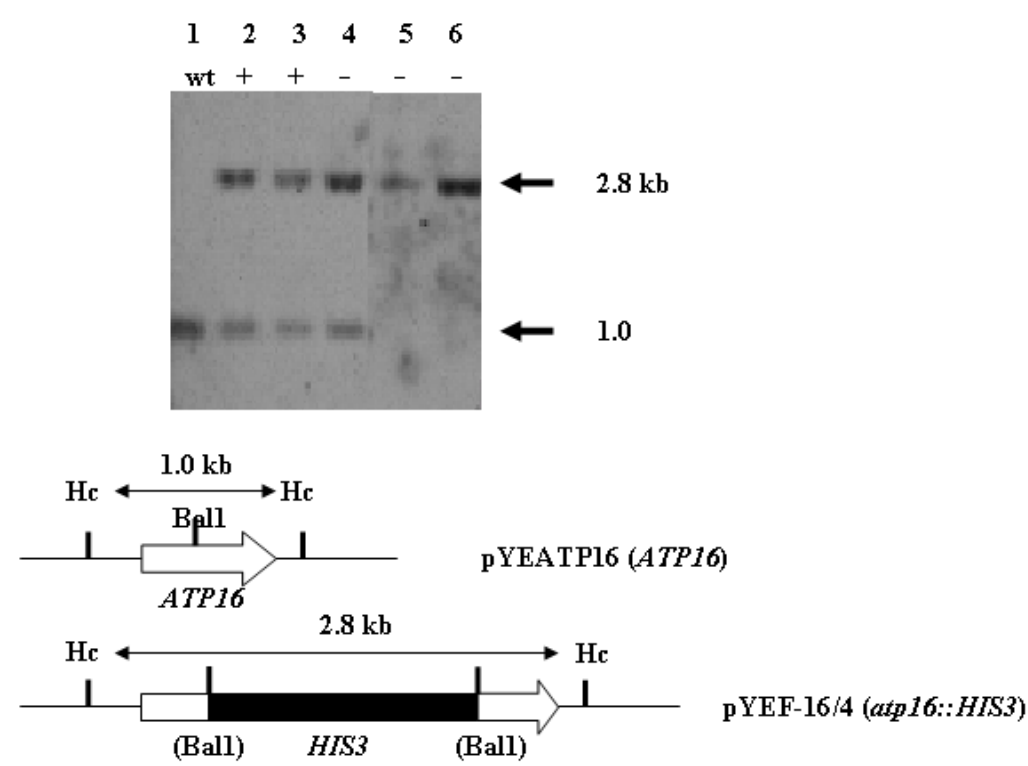

Figure 1. Southern hybridization of genomic DNA isolated from ATP16-disruptants with HIS3

Various yeast strains, DC5, LL20, W303-1A and W303-1B were used in the experiments. The total DNA (genomic DNA) was isolated from the yeast strains and digested with HincII. After the digestion the genomic DNA was applied to a $0.8 \%$ agarose gel electrophoresis, blotted on to a nylon membrane and then hybridized to DIG-labeled ATP16. Lower panel showed the plasmids containing ATP16 (pYEATP16), and ATP16 disrupted with HIS3 (pYEF-16/4) using the homologous recombination described in Materials and Methods.

Lane1, W303-1B (WT, ATP16a, ATP16b, Gly+); lanes 2, W30 (atp16a::HIS3, ATP16b, Gly+); lane 3, W3 (atp16a::HIS3, ATP16b, Gly+) lane 4, W202 (ATP16a, atp16b::HIS3, Gly-); lane 5, W19 (atp16a::HIS3. Atp16b::HIS3, Gly-); lane 6, W6 (atp16a::HIS3, atp16b::HIS3, Gly-). The disrupted ATP16 with HIS3 was determined by the Southern hybridization of the Sph1-digested genomic DNA used HIS3 as a probe. 
(a) 1 st pobe: $A T P 16$

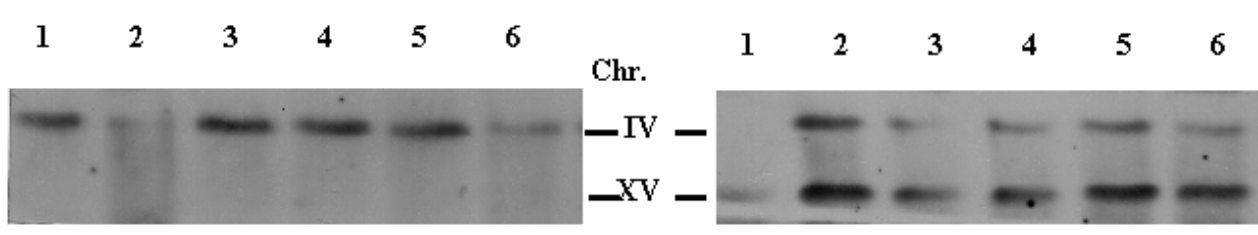

Figure 2. Chromosome Southern hybridization of ATP16 disruptants

Chromosomes were isolated and electrophoresed in $1 \%$ agarose gel on a CHEF apparatus, blotted onto a nylon membrane and then hybridized successively to DIG-labeled ATP16 (a) and HIS3 (b). Electrophoresis was performed for $34 \mathrm{~h}$ with a switching interval of $55 \mathrm{~s}$ for $17 \mathrm{~h}$ and $90 \mathrm{~s}$ for $17 \mathrm{~h}(1-4)$. Lane 1, W303-1B (WT, ATP16a, ATP16B); lane 2, W30 (atp16a::HIS3, ATP16b, Gly+); lane 3, W3 (atp16a::HIS3, ATP16b, Gly+); lane 4, W202 (ATP16a, atp16b::HIS3, Gly-); lane 5, W19 (atp16a::HIS3. Atp16b::HIS3, Gly-); lane 6, W6 (atp16a::HIS3, atp16b::HIS3, Gly-).

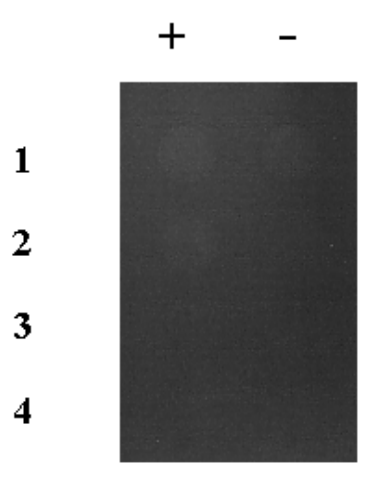

$24 h$

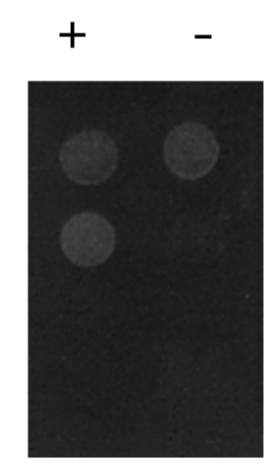

48h

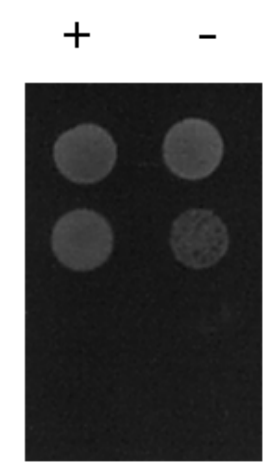

$72 h$

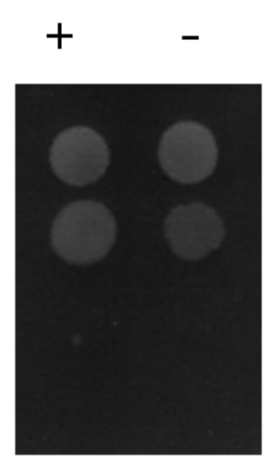

96h

Figure 3. Glycerol phenotype of ATP16 disruptants

Various ATP16-disruptants were counted $\left(10^{6} / \mathrm{ml}\right)$ and grown on a glycerol medium (YPG) for 24, 48, 72, and 96 $\mathrm{h}$ at $30^{\circ} \mathrm{C}$. Top (1), W0303-1B (WT, ATP16a, ATP16b); second (2), W30 (atp16a::HIS3, ATP16b); third (3), W202 (ATP16a, atp16b::HIS3); bottom (4), W19 (atp16a::HIS3, atp16b::HIS3).

In each dish at left $(+)$ are the wild-type ATP16 (YDL004w) gene with multi-copy plasmid (pYEATP16) added to the laboratory strain and the ATP16 disruptants. The dishes at right (-) are without the added wild-type ATP16 (YDL004w) gene (only the control vector, pAML20 was added). 
(a) probe: $A T P 16$

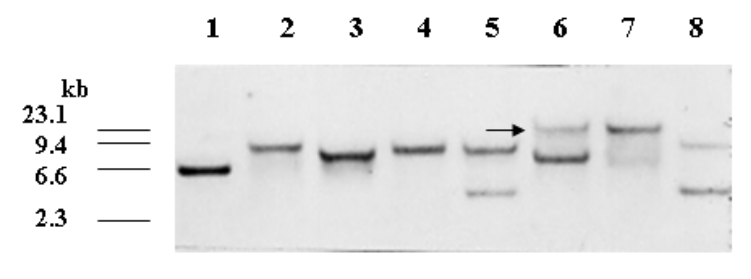

(b) probe: CEN4 or $N T H 1$

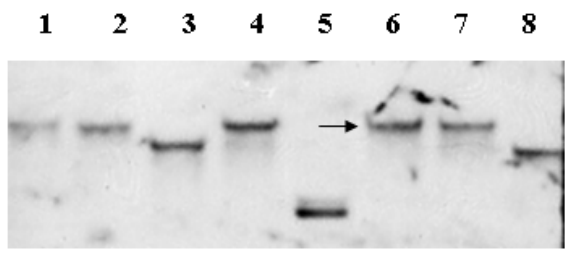

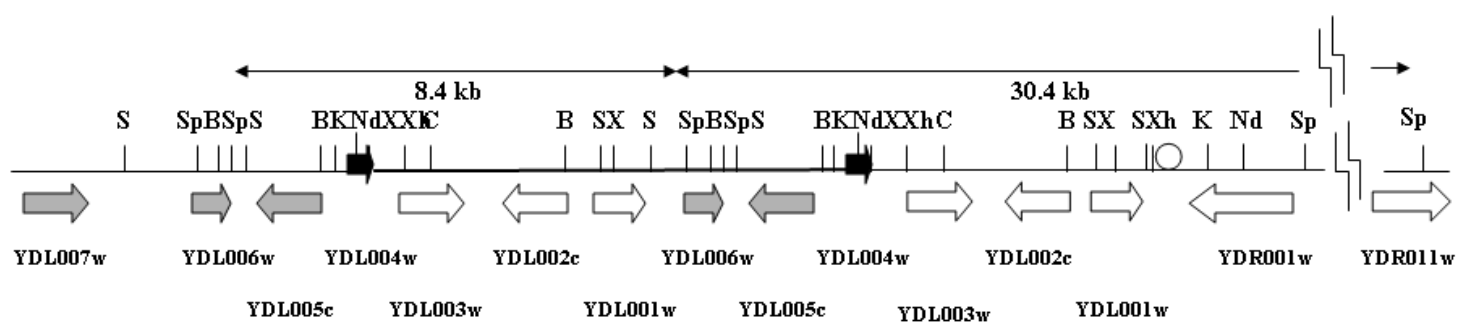

Figure 4. Analyses of DNA region between ATP16a and ATP16b

The total (genomic) DNAs from laboratory strains W303-1A, W303-1B, or S288C were isolated and digested with different restriction enzymes and applied to a $0.8 \%$ agarose gel. After electrophoresis, the digestive DNA fragments were blotted onto a nylon membrane and then hybridized with DIG-labeled ATP16 (a) and CEN4 and NTH1. Lane 1, BamHI; lane 2, ClaI; lane 3, KpnI; lane 4, XhoI; lane 5, NdeI; lane 6, SphI; lane 7, SphI/NcoI; lane $8, X b a \mathrm{I}$. Arrow indicated the $30.4 \mathrm{~kb}$ fragment.

B, BamHI; C, ClaI; K, KpnI; N, NcoI; Nd, NdeI; S, ScaI; Sp, SphI; X, Xba; Xh XhoI.

Chromosome IV, left arm.

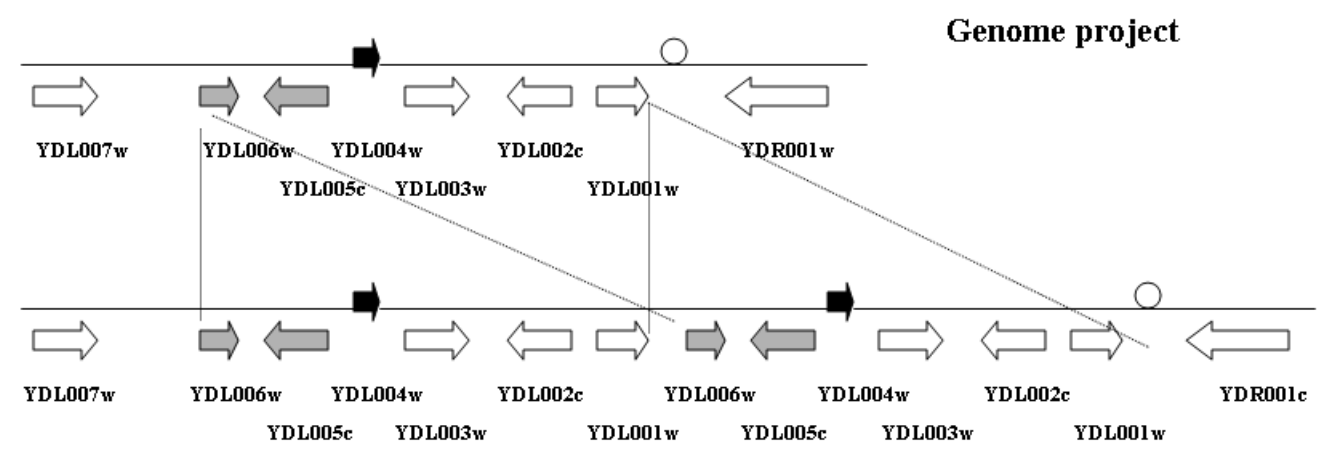

This study $\overline{1 \mathrm{~kb}}$

:CEN4

Figure 5. Map of two ATP16s and neighboring ORFs

ORFs on chromosome IV were taken as systematic designations in the S.cerevisiae genome.

Black arrow, ATP16 (YDL004w); gray arrow, upstream ORFs (YDL006w and YDL005w); white arrow, downstream ORFs (YDL003w, YDL002c, and YDL001w) of ATP16 (YDL004w, mips, http://mips.gsf.de/). 

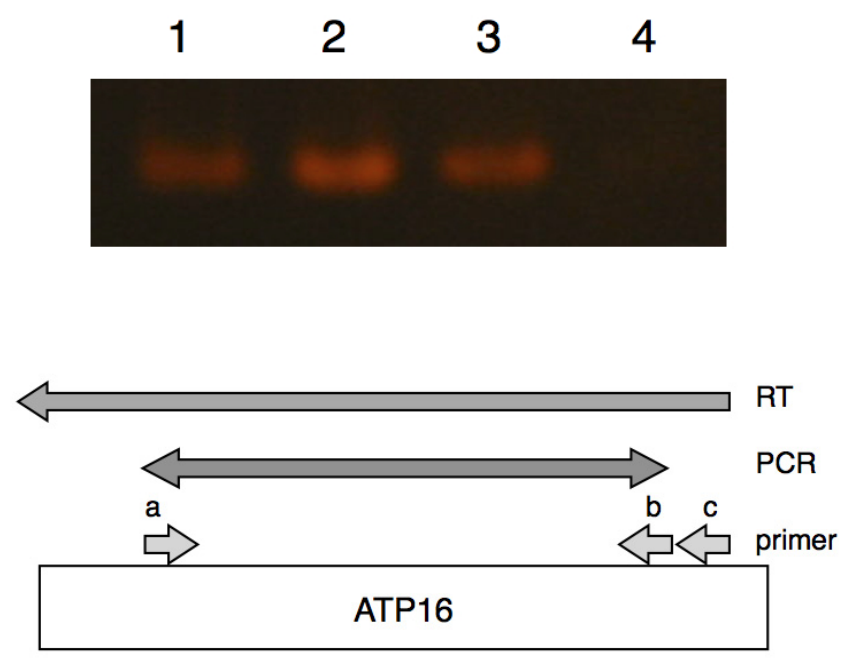

Figure 6. Transcripts of ATP16a and ATP16b

The RT-PCR experiments were carried out to see the transcripts of both ATP16a and ATP16b using the sense (primer a) and anti-sense primers (primers b or c) in the lower panel. The procedures of the RT-PCR experiments were described in Materials and Methods. The upper panel was shown the products of the RT-PCR. Lane 1, W303-1B (WT, ATP16a, ATP16b); lane 2, W30 (atp16a::HIS3, ATP16b); lane 3, W202 (ATP16a, atp16b::HIS3); lane 4, W19 (atp16a::HIS3, atp16b::HIS3). 